\title{
Finite conductivity minimum in bilayer graphene without charge inhomogeneities
}

\author{
Maxim Trushin, ${ }^{1,2}$ Janik Kailasvuori, ${ }^{3}$ John Schliemann, ${ }^{2}$ and A. H. MacDonald ${ }^{1}$ \\ ${ }^{1}$ Physics Department, University of Texas, 1 University Station C1600, Austin, 78712 Texas, USA \\ ${ }^{2}$ Institut für Theoretische Physik, Universität Regensburg, 93040 Regensburg, Germany \\ ${ }^{3}$ Max-Planck-Institut für Physik komplexer Systeme, Nöthnitzer Str. 38, 01189 Dresden, Germany
}

(Received 22 February 2010; revised manuscript received 15 September 2010; published 5 October 2010)

\begin{abstract}
Boltzmann transport theory fails near the linear band crossing of single-layer graphene and near the quadratic band crossing of bilayer graphene. We report on a numerical study which assesses the role of interband coherence in transport when the Fermi level lies near the band-crossing energy of bilayer graphene. We find that interband coherence enhances conduction, and that it plays an essential role in bilayer graphene's minimum conductivity phenomena. This behavior is qualitatively captured by an approximate theory which treats interband coherence in a relaxation-time approximation. On the basis of this short-range-disorder model study, we conclude that electron-hole puddle formation is not a necessary condition for finite conductivity in bilayer graphene at zero average carrier density.
\end{abstract}

DOI: $10.1103 /$ PhysRevB.82.155308

PACS number(s): $72.80 . \mathrm{Vp}$

\section{INTRODUCTION}

The robust conductivity of nearly neutral graphene sheets ${ }^{1,2}$ is interesting from a theoretical point of view, awkward ${ }^{3}$ for some potential applications, and among the most unexpected of graphene transport study discoveries. As a function of ambipolar carrier density the minimum conductivity is $\sim e^{2} / h$ with relatively small sample to sample variation. The generally accepted explanation ${ }^{4-12}$ for this property starts by recognizing the influence of randomly distributed charged impurities ${ }^{13,14}$ which induce electron-hole puddles ${ }^{15,16}$ in graphene when the global average carrier density is low. Partly because of ${ }^{5}$ the role of Klein tunneling in Dirac-type systems, a network of conducting puddles can account for global conduction that remains finite when the average carrier concentration falls to zero. There are, however, indications that this explanation is incomplete. In particular, suspended graphene ${ }^{17-19}$ samples still exhibit a minimum conductivity even though charged impurities appear to be removed upon annealing and puddle formation should therefore be suppressed. The present work is motivated by the view that graphene's minimum conductivity phenomena are more general than sometimes thought, and not necessarily associated with smooth inhomogeneities.

Independent of disorder character, transport near the band-crossing energies of graphene systems differs from transport near typical semiconductor band extrema in three important ways: (i) the absence of an energy gap between the conduction and valence bands, (ii) the peculiar momentum dependence of intersublattice hopping in graphene systems that leads to the Dirac-type electronic structure, and (iii) in the case of single-layer graphene the linear band dispersion which causes the two-dimensional density of states to vanish in the absence of disorder. The goal of this paper is to shed light on which of these aspects is responsible for conductivity minimum phenomena. Since experiment indicates that there is no essential difference between the minimum conductivity behavior of single and bilayer cases, the dispersion law does not appear to play an essential role. The minimum conductivity is also finite in suspended bilayer graphen $\mathrm{e}^{20}$ samples, even though the charge carriers in this case exhibit the same parabolic ${ }^{21}$ dispersion that is found in conventional two-dimensional electron systems. We therefore focus on bilayers, and on the role of momentum-sublattice coupling in the absence of an energy gap. This problem has received relatively little theoretical attention. ${ }^{22-30}$

Momentum-sublattice coupling in bilayers is well described by the $\pi$-band envelope-function effective band Hamiltonian, ${ }^{1,21}$

$$
H_{0}=-\frac{\hbar^{2}}{2 m}\left(\begin{array}{cc}
0 & \left(k_{x}-i k_{y}\right)^{2} \\
\left(k_{x}+i k_{y}\right)^{2} & 0
\end{array}\right) .
$$

Here $m \simeq 0.05 m_{0}$ is the effective mass, $m_{0}$ is the bare electron mass, $\mathbf{k}$ is the two-component particle momentum, and the matrix structure originates from the layer and sublattice degrees of freedom. The Hamiltonian $H_{0}$ does not contain the trigonal warping term and just represents the minimal model where the conductivity minimum does not vanish. The spectrum of $H_{0}$ consists of parabolic conduction and valence bands that touch at eigenenergy $E=0$. The sublattice degree of freedom is frequently viewed as a pseudospin in order to exploit analogies between spin-orbit and pseudospin-orbit coupling. From this point of view $H_{0}$ can be considered as expressing an effective Zeeman coupling to pseudospins that has a strength $\hbar \Omega_{k}=\hbar^{2} k^{2} / m$ which is momentum-magnitude dependent, and a $\hat{x}-\hat{y}$ plane orientation angle $\phi=2 \phi_{\mathrm{k}}$, where $\phi_{\mathbf{k}}$ is the two-dimensional momentum direction. The pseudospin precession axis therefore changes whenever an electron is scattered between momentum states. When the precession frequency $\Omega_{k}$ is larger than the momentum scattering rate $\tau^{-1}$, the pseudospin precesses a few times between collisions and any initial transverse component is likely to be randomized. The conductance minima phenomena occur for energies $E$ near zero for which $\Omega_{k} \tau$ is always small and pseudospin components transverse to the precession axis are not expected to randomize. This observation alone suggests the possibility that atypical quantum effects could play a role. This is what we can see in Fig. 1: the conductivity never falls to zero for any reasonable choice of parameters as long 


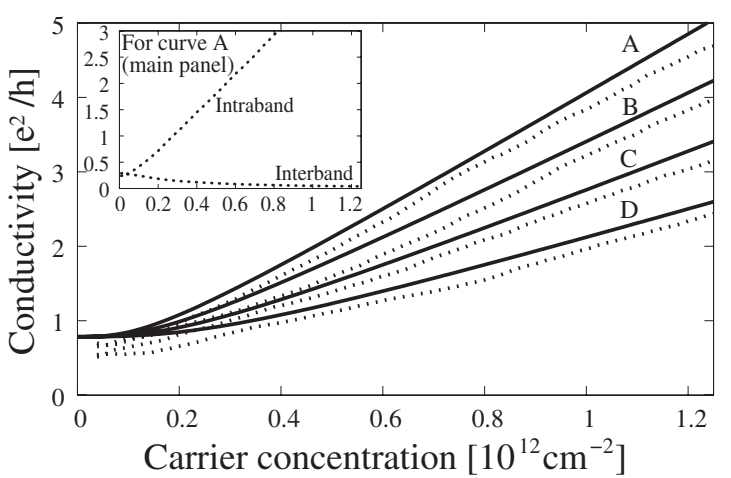

FIG. 1. The dotted curves depict the electrical conductivity of bilayer graphene (per spin/valley) as a function of carrier concentration computed according to the Kubo formula (2) for the series of model parameters specified in Table I. The solid lines correspond to the analytical approximation which is the sum of the Drude conductivity $\sigma_{\mathrm{D}}$ and an interband coherent correction $\Delta \sigma$ given by Eq. (5). The inset illustrates the decomposition of the conductivity for disorder model A into intraband and interband coherent contributions proportional, respectively, to the intraband and interband terms in the velocity operator in Eq. (2).

as the interband coherence is included in the model, even when charged impurities are absent and electron-hole puddle formation ${ }^{6,12}$ is not expected. We focus solely on the zerotemperature limit. The finite temperature $e^{6,11,31}$ can lead to the thermally excited carriers which may spoil the interband coherence effect. The intervalley scattering is also assumed to be absent here.

\section{KUBO AND BOLTZMANN THEORIES}

We have evaluated the conductivity numerically using the noninteracting particle Kubo formula expression. This approach has the advantage that it is exact, ${ }^{32}$ or at least would be if computational resources were infinite. On the other hand it does not lend itself to a satisfying qualitative understanding. We therefore compare our numerical results with those predicted by a heuristic semiclassical theory ${ }^{33,34}$ that captures interband coherence corrections to the Boltzmann equation. We first comment briefly on these two approaches.

The finite-size Kubo formula for the static conductivity is

$$
\sigma_{\mathrm{K}}=-\frac{i \hbar e^{2}}{L^{2}} \sum_{n, n^{\prime}} \frac{f_{E_{n}}^{0}-f_{E_{n^{\prime}}}^{0}}{E_{n}-E_{n^{\prime}}} \frac{\left\langle n\left|v_{x}\right| n^{\prime}\right\rangle\left\langle n^{\prime}\left|v_{x}\right| n\right\rangle}{E_{n}-E_{n^{\prime}}+i \eta},
$$

where $\mathbf{v}$ is the velocity operator, $f_{E_{n}}^{0}$ is the Fermi-Dirac distribution function, and $|n\rangle$ denotes an exact eigenstate of the Schrödinger equation for a finite-size disordered system with periodic boundary conditions: $\left(H_{0}+U\right) \psi_{n}=E_{n} \psi_{n}$ with $U(\mathbf{r})$ $=u_{0} \Sigma_{i}^{N_{s}} \delta\left(\mathbf{r}-\mathbf{R}_{i}\right)$ for the short-range-disorder model we consider. The scattering locations $\mathbf{R}_{i}$ and potential signs are random. We solve the Schrödinger equation using a large momentum-space cutoff $k^{*} \approx \sqrt{5 \times 10^{13}} \mathrm{~cm}^{-1}$ which corresponds to the energy scale at which the split-off bands of bilayer graphene become relevant and our two-band model no longer applies.
The physical dc conductivity can be obtained from Eq. (2) by extracting the limit in which the system size first approaches $\infty$ and then $\eta$ approaches zero maintaining a value larger than the typical level spacing $\delta E$. For the model considered here $\delta E=\left(2 \pi \hbar^{2}\right) /\left(m L^{2}\right)$, where $L^{2}$ is the finite-size system area. The finite value of $\eta$ can be understood as representing energy uncertainty due to the finite lifetime of electrons in a system coupled to source and drain reservoirs. To eliminate the influence of the bath on the conductivity itself, the momentum relaxation time $\tau$ due to internal scatterers must be much smaller than $\hbar / \eta{ }^{35}$ We estimate $\tau$ using the Fermi golden-rule expression: $\tau=2 \hbar^{3} / m n_{s} u_{0}^{2}$, where $n_{s}$ $=N_{s} / L^{2}$ is the impurity density. Since the smallest possible $\delta E$ is limited by numerical practicalities, we can estimate the conductivity only for relatively strong disorder. Conductivities obtained directly from Eq. (2) undergo the phasecoherent fluctuations; we simulate macroscopic system conductivities by averaging the conductivity over an energy interval containing 10-100 levels, over boundary conditions, and over several disorder potential realizations. Note that the conductivity fluctuation amplitude turns out to be essentially smaller that $e^{2} / h$ near the neutrality point. This makes our numerical approach reliable for the conductivity minimum evaluation.

Below we compare our numerical results for the conductivity to an analytic modified Boltzmann equation theory. When coherence effects are retained the distribution function $f(\mathbf{k})$ becomes a $2 \times 2$ matrix with band labels. The steadystate limit of its equation of motion is

$$
\frac{1}{\hbar}\left\{e \mathbf{E} \frac{\partial f(\mathbf{k})}{\partial \mathbf{k}}+i\left[H_{0}, f(\mathbf{k})\right]\right\}=I[f(\mathbf{k})],
$$

where $\mathbf{E}$ is an electric field small enough to justify linearresponse theory, $I[f(\mathbf{k})]$ is the collision integral which accounts for disorder scattering, and the commutator $\left[H_{0}, f(\mathbf{k})\right]$ accounts for the difference in time evolution between conduction- and valence-band eigenstates. When the collision integral is evaluated to leading (second) order in the (configuration-averaged) impurity potential, the collision term (including its off-shell terms ${ }^{34,36}$ ) reduces to the simple matrix relation-time form, $I[f(\mathbf{k})] \rightarrow-f^{(1)}(\mathbf{k}) / \tau$, where $f^{(1)}$ is the deviation from equilibrium. This is a remarkable property of the two-band bilayer model with $\delta$-function scatterers. In the $H_{0}$ eigenstate basis, the density-matrix linear response $f^{(1)}$ then reads

$$
f^{(1)}=\tau e \mathbf{E}\left(\begin{array}{cc}
\mathbf{v}_{++}\left(-\frac{\partial f_{E_{k+}}^{0}}{\partial E_{k+}}\right) & \mathbf{v}_{+-} \frac{f_{E_{k-}}^{0}-f_{E_{k+}}^{0}}{\hbar \Omega_{k}\left(1+i \Omega_{k} \tau\right)} \\
\mathbf{v}_{-+} \frac{f_{E_{k-}}^{0}-f_{E_{k+}}^{0}}{\hbar \Omega_{k}\left(1-i \Omega_{k} \tau\right)} & \mathbf{v}_{--}\left(-\frac{\partial f_{E_{k-}}^{0}}{\partial E_{k-}}\right)
\end{array}\right) .
$$

Here, $E_{k \pm}$ are the eigenvalues of $H_{0}, \Omega_{k}=\hbar k^{2} / m$, and $\mathbf{v}_{\sigma, \sigma^{\prime}}$ is the velocity operator written in the $H_{0}$ eigenstate basis. Given this approximation for the linear response of the distribution function, it is easy to calculate the Boltzmann conductivity: $\sigma_{\mathrm{B}}=j_{x} / E_{x}$, where $j_{x}$ is the electrical current, $\mathbf{j}$ $=e \int \frac{d^{2} k}{(2 \pi)^{2}} \operatorname{Tr}\left[\mathbf{v} f^{(1)}(\mathbf{k})\right]$. Note that neither $\mathbf{v}$ nor $f^{(1)}(\mathbf{k})$ are diag- 
TABLE I. Parameters for Fig. 1: $\tau$ is the momentum relaxation time, $\mu=e \tau / m$ is the mobility of carriers, $n_{s}$ is the concentration of short-range scatterers with strength fixed at a value $u_{0}=\pi^{2} \hbar^{2} / 5 m$ small enough to validate the golden-rule lifetime expression, and $\delta E=2 \pi \hbar^{2} / L^{2} m$ is the level spacing for sample size $L=1.8$ $\times 10^{-5} \mathrm{~cm}$. At this sample size dependence on $L$ is weak. The momentum cutoff $k^{*}$ and $L$ fix the Hamiltonian matrix dimension at $3362 \times 3362$. The computations have been performed at zero temperature.

\begin{tabular}{|c|c|c|c|c|}
\hline Label & $\begin{array}{c}\tau \\
\left(10^{-13} \mathrm{~s}\right)\end{array}$ & $\begin{array}{l}\mu \\
\left.\mathrm{cm}^{2} / \mathrm{V} \mathrm{s}\right)\end{array}$ & $\begin{array}{c}n_{s} \\
\left(10^{12} \mathrm{~cm}^{-2}\right)\end{array}$ & $\eta \tau / \hbar$ at $\eta=10 \delta E$ \\
\hline A & 0.30 & 1 & 0.81 & 0.13 \\
\hline B & 0.25 & 0.83 & 0.97 & 0.10 \\
\hline $\mathrm{C}$ & 0.20 & 0.66 & 1.22 & 0.08 \\
\hline $\mathrm{D}$ & 0.15 & 0.50 & 1.62 & 0.06 \\
\hline
\end{tabular}

onal, and that $\mathbf{j}$ therefore includes interband coherence contributions. The intraband contribution to the conductivity stems from the diagonal terms in Eq. (4) and is given by the simple Drude formula $\sigma_{\mathrm{D}}=e^{2} n \tau / m$, where $n$ is the carrier concentration $n=k_{F}^{2} /(4 \pi)$ with $k_{F}$ being the Fermi momentum.

\section{RESULTS}

Numerical results for the dependence of Kubo conductivity on carrier density are presented in Fig. 1 for a series of model parameter values summarized in Table I. Our main finding is that the conductivity remains finite as the carrier density approaches zero. We do not observe any systematic dependence of the minimum conductivity, $\sigma_{\min } \sim 0.7 e^{2} / h$ per spin and valley, on model system parameters.

There are two elements in our model which couple the two bands in Hamiltonian (1) and both are important for the conductivity minimum phenomena. The first is the velocity operator $\mathbf{v}_{\sigma, \sigma^{\prime}}$. The second is the scattering potential $U(\mathbf{r})$ which can produce interband scattering. We quantify the role of interband coupling by separating both velocity operators in Eq. (2) into intraband and interband contributions to express the conductivity as the sum of intraband $\left(\propto \mathbf{v}_{ \pm \pm} \mathbf{v}_{ \pm \pm}\right)$, interband $\left(\propto \mathbf{v}_{ \pm \mp} \mathbf{v}_{\bar{\mp}}\right)$, and interference $\left(\propto \mathbf{v}_{ \pm \pm} \mathbf{v}_{ \pm \mp}\right)$ terms. We find that the interference terms average to negligible values. As illustrated in Fig. 1 (inset), the intraband contribution dominates in the higher carrier-density Boltzmann transport regime, as expected. However, it does not completely vanish at zero density as long as the scattering potential is in play. The interband contribution, in contrast, increases substantially near the neutrality point. Figure 1 (inset) shows that $\sigma_{\min }$ is due substantially, and possibly dominantly, to the nonclassical interband coherent contribution.

In an attempt to isolate the source of the peculiar conductivity behavior we have in Fig. 2 compared the numerical conductivities of our bilayer model with those of a decoupled band model in which $H_{0} \rightarrow \hbar^{2}\left(k_{x}^{2}+k_{y}^{2}\right) \sigma_{z} / 2 \mathrm{~m}$. The two models have the same density of states, but the decoupled band model has no interband velocity-operator matrix elements,
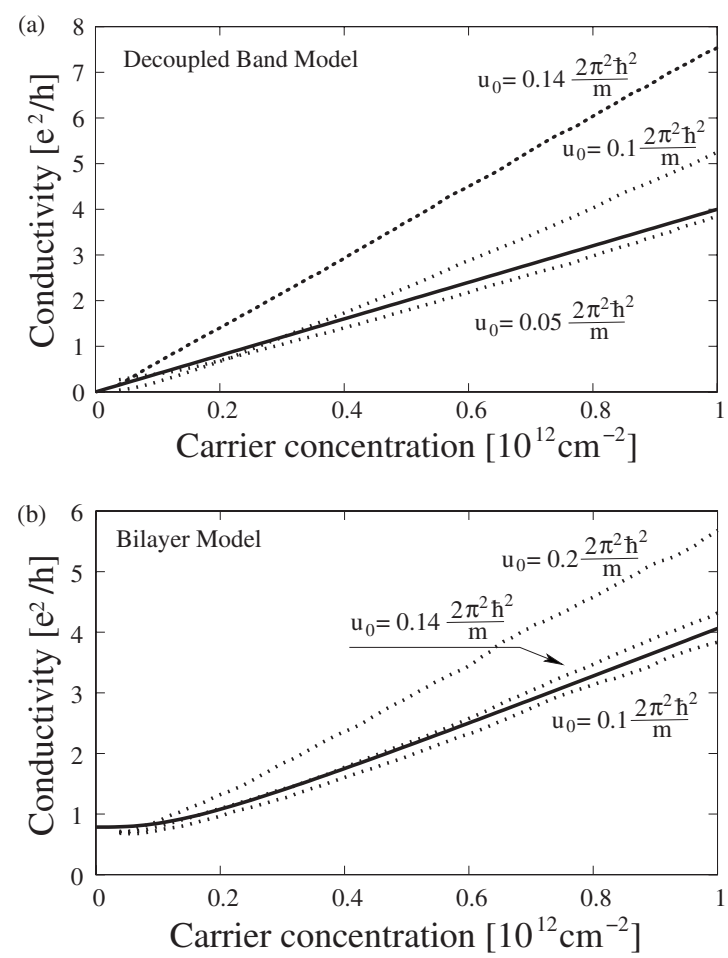

FIG. 2. Comparison between Kubo conductivities, Eq. (2), of (a) the decoupled band model and (b) bilayer graphene. These results were obtained for a series of models with identical golden-rule relaxation times $\tau=0.3 \times 10^{-13} \mathrm{~s}$, and sample sizes $L=1.8$ $\times 10^{-5} \mathrm{~cm}$. (The concentration of scatterers $n_{s}$ was adjusted appropriately in each case.) One can see that the conductivity minimum for the decoupled band model vanishes whereas for the bilayer model it is finite and insensitive to the scattering potential strength. The thick solid lines show the naive prediction of (a) Drude theory and (b) our interband coherent Boltzmann model with golden-rule relaxation times.

and the scattering potential $U(\mathbf{r})$ is not able to couple the bands. The golden-rule relaxation times of the models are identical when we also let $u_{0} \rightarrow u_{0} / \sqrt{2}$ to compensate for the suppression of right-angle scattering in the bilayer case. Figure 2(a) shows that $\sigma_{\min } \rightarrow 0$ in the decoupled band model. Deviations from the Drude formula at low carrier concentrations in Fig. 2(a) have a negative sign and are consistent with Anderson insulator behavior. In Fig. 2 we also see enhanced conductivity compared to the Boltzmann model at larger values of $u_{0}$ at high carrier densities, which we attribute simply to an overestimate of scattering rates by the golden-rule expression. The small negative deviation from the Boltzmann model at small $u_{0}$ may partially reflect weak localization. ${ }^{27,28}$

In the zero-temperature limit of the generalized Boltzmann theory, the integrals over wave vector in the expression for the interband-coherence conductivity can be evaluated to obtain

$$
\Delta \sigma=\frac{e^{2}}{2 h}\left[\frac{\pi}{2}-\tan ^{-1}\left(\Omega_{k_{F}} \tau\right)\right]
$$

and the total Boltzmann conductivity will be $\sigma_{\mathrm{B}}=\sigma_{\mathrm{D}}+\Delta \sigma$. It follows that $\sigma_{\mathrm{B}}$ never falls down below $\sigma_{\min }=\pi e^{2} / 4 h$ for any 
choice of parameter values. This value agrees with Ref. 30, where a related modified Boltzmann approach is combined with a four-band effective Hamiltonian for the carriers, as well as with recent theoretical predictions ${ }^{36}$ using other closely related approaches. Our $\sigma_{\min }$ differs from the one obtained for ballistic bilayer graphene, ${ }^{24,25,37}$ where the $\sigma_{\min }$ is attributed to evanescent modes penetrating the sample from contacts. We emphasize that Eq. (5) should be only seen as the rough analytical approximation for our numerical results. Equation (5) together with the Drude term fits the numerical conductivity curves quite well but it does not mean that the conductivity minimum is exactly $\pi e^{2} / 4 h$. However, the similarity of $\sigma_{\text {min }}$ values obtained with different approximate approaches might suggest a common underlying origin in a relationship to the spectral flows associated with the topological properties ${ }^{26,38,39}$ of graphene singlelayer and bilayer bands.

To conclude, in our approximate theory the minimum conductivity is mainly due to a electric field driven coherence between the conduction and valence bands. Momentum space drift due to the electric field does not repopulate momenta in a full valence band, as maintained in textbook transport theory, but it does drive the system from equilibrium in that it alters the relationship between momentum and sublattice pseudospin. There is still exactly one electron at each momentum but the momentum states no longer come with definite helicity, i.e., are no longer the equilibrium valence-band wave functions. As consequence, all valence electrons contribute to the conductivity, although the contribution from large momenta $\left(\Omega_{k} \tau>1\right)$ gets suppressed by the larger spin precession. Our numerical calculation provides at least partial support for this picture. Quantitative discrepancies might come from not accounting for weak localization effects and the influence of disorder on the equilibrium state.

\section{SUMMARY}

We have used numerical exact-diagonalization calculations to demonstrate (i) that the conductivity of bilayer graphene in the limit of zero carrier density $\sigma_{\min } \sim e^{2} / h$, (ii) that interband coherence response plays a key role in this property, and (iii) that the formation of electron-hole puddles due to strong but smooth potential variations is not a necessary condition for the minimum conductivity phenomena. We believe that our model is relevant to suspended graphene samples in which charged impurities are removed by annealing. When spin and valley degeneracy is accounted for we estimate numerically $\sigma_{\min }^{-1} \sim 8.2 \mathrm{k} \Omega$ which appears to be consistent with current measurements. ${ }^{20}$

\section{ACKNOWLEDGMENTS}

This work was funded by DFG through the Project No. TR 1019/1-1 (M.T.). J.S. was supported by DFG via Grant No. GRK 1570. A.H.M. was supported by the Welch Foundation (Grant No. F-1473) and by the NSF-DMR program.
${ }^{1}$ A. K. Geim and K. S. Novoselov, Nature Mater. 6, 183 (2007).

${ }^{2}$ A. H. Castro Neto, F. Guinea, N. M. R. Peres, K. S. Novoselov, and A. K. Geim, Rev. Mod. Phys. 81, 109 (2009).

${ }^{3}$ A. K. Geim, Science 324, 1530 (2009).

${ }^{4}$ S. Adam, E. H. Hwang, V. M. Galitski, and S. Das Sarma, Proc. Natl. Acad. Sci. U.S.A. 104, 18392 (2007).

${ }^{5}$ V. V. Cheianov, V. I. Fal'ko, B. L. Altshuler, and I. L. Aleiner, Phys. Rev. Lett. 99, 176801 (2007).

${ }^{6}$ S. Adam and S. Das Sarma, Phys. Rev. B 77, 115436 (2008).

${ }^{7}$ E. Rossi, S. Adam, and S. Das Sarma, Phys. Rev. B 79, 245423 (2009).

${ }^{8}$ E. Rossi and S. Das Sarma, Phys. Rev. Lett. 101, 166803 (2008).

${ }^{9}$ M. M. Fogler, Phys. Rev. Lett. 103, 236801 (2009).

${ }^{10}$ M. M. Fogler, D. S. Novikov, and B. I. Shklovskii, Phys. Rev. B 76, 233402 (2007).

${ }^{11}$ S. Adam and M. D. Stiles, Phys. Rev. B 82, 075423 (2010).

${ }^{12}$ S. Das Sarma, E. H. Hwang, and E. Rossi, Phys. Rev. B 81, 161407 (2010).

${ }^{13}$ K. Nomura and A. H. MacDonald, Phys. Rev. Lett. 96, 256602 (2006).

${ }^{14}$ T. Ando, J. Phys. Soc. Jpn. 75, 074716 (2006).

${ }^{15}$ J. Martin, N. Akerman, G. Ulbricht, T. Lohmann, J. H. Smet, K. von Klitzing, and A. Yacoby, Nat. Phys. 4, 144 (2008).

${ }^{16}$ Y. Zhang, V. W. Brar, C. Girit, A. Zettl, and M. F. Crommie, Nat. Phys. 5, 722 (2009).

${ }^{17}$ X. Du, I. Skachko, A. Barker, and E. Y. Andrei, Nat. Nanotechnol. 3, 491 (2008).
${ }^{18}$ K. Bolotin, K. Sikes, Z. Jiang, M. Klima, G. Fudenberg, J. Hone, P. Kim, and H. Stormer, Solid State Commun. 146, 351 (2008).

${ }^{19}$ K. I. Bolotin, K. J. Sikes, J. Hone, H. L. Stormer, and P. Kim, Phys. Rev. Lett. 101, 096802 (2008).

${ }^{20}$ B. E. Feldman, J. Martin, and A. Yacoby, Nat. Phys. 5, 889 (2009).

${ }^{21}$ E. McCann and V. I. Falko, Phys. Rev. Lett. 96, 086805 (2006).

${ }^{22}$ J. Nilsson, A. H. Castro Neto, F. Guinea, and N. M. R. Peres, Phys. Rev. Lett. 97, 266801 (2006).

${ }^{23}$ M. Koshino and T. Ando, Phys. Rev. B 73, 245403 (2006).

${ }^{24}$ M. Katsnelson, Eur. Phys. J. B 52, 151 (2006).

${ }^{25}$ I. Snyman and C. W. J. Beenakker, Phys. Rev. B 75, 045322 (2007).

${ }^{26}$ J. Cserti, A. Csordás, and G. Dávid, Phys. Rev. Lett. 99, 066802 (2007).

${ }^{27}$ R. V. Gorbachev, F. V. Tikhonenko, A. S. Mayorov, D. W. Horsell, and A. K. Savchenko, Phys. Rev. Lett. 98, 176805 (2007)

${ }^{28}$ K. Kechedzhi, V. I. Falko, E. McCann, and B. L. Altshuler, Phys. Rev. Lett. 98, 176806 (2007).

${ }^{29}$ A. G. Moghaddam and M. Zareyan, Phys. Rev. B 79, 073401 (2009).

${ }^{30}$ D. Culcer and R. Winkler, Phys. Rev. B 79, 165422 (2009).

${ }^{31}$ M. Lv and S. Wan, Phys. Rev. B 81, 195409 (2010).

${ }^{32}$ K. Nomura and A. H. MacDonald, Phys. Rev. Lett. 98, 076602 (2007). 
${ }^{33}$ M. Trushin and J. Schliemann, Phys. Rev. Lett. 99, 216602 (2007).

${ }^{34}$ M. Auslender and M. I. Katsnelson, Phys. Rev. B 76, 235425 (2007).

${ }^{35}$ D. C. Licciardello and D. J. Thouless, J. Phys. C 8, 4157 (1975).

${ }^{36}$ J. Kailasvuori and M. C. Lüffe, J. Stat. Mech.: Theory Exp.
(2010) P06024.

${ }^{37}$ J. Cserti, Phys. Rev. B 75, 033405 (2007).

${ }^{38}$ K. Nomura, M. Koshino, and S. Ryu, Phys. Rev. Lett. 99, 146806 (2007).

${ }^{39}$ J. H. Bardarson, J. Tworzydło, P. W. Brouwer, and C. W. J. Beenakker, Phys. Rev. Lett. 99, 106801 (2007). 